\title{
Asymptotic Properties of Fractional Delay Differential Equations
}

\author{
Katja Krol* \\ Humboldt Universität zu Berlin
}

March 22, 2009

\begin{abstract}
In this paper we study the asymptotic properties of $d$-dimensional linear fractional differential equations with time delay. First results on existence and uniqueness of solutions are presented. Then we propose necessary and sufficient conditions for asymptotic stability of equations of this type using the inverse Laplace transform method.
\end{abstract}

Key words: fractional differential equations, delay differential equation, linear equations, existence, uniqueness, asymptotic stability, Laplace transform

AMS subject classification: primary 34K06, secondary 26A33, 34K20, 34K25, 34D05, 34A12

\section{Introduction}

Delay or functional differential equations (DDEs) are used to describe systems with time delay. Such processes arise in many areas of science - in biology (time to maturity and incubation time), controlled systems (delayed feedback), economics (time to transport, time lag for getting information). In general, time delay is believed to have a negative impact on stability of systems. Detailed results on asymptotic properties of DDEs can be found in the book of J.K. Hale and S.M. Verduyn Lunel, [6]. The general linear case is treated in [8]. Over the past years the theory of fractional order linear delay differential equations (FDDEs) has attracted attention of mathematicians and engineers. In [1], [13], [5] the authors discuss the analytical stability bound for the class of fractional delay difference equations. In [9], [10] finite time stability of robotic systems is studied, where a time delay appears in

\footnotetext{
${ }^{*}$ This work was supported by the Deutsche Telekom Stiftung and the International Research Training Group 1339 SMCP.
} 
$P D^{\alpha}$ fractional control system.

However, there exists no general theory of equations of such a type. In this paper we study a generalization of equations presented in [1], [13], [5], [9], [10], [14]. We consider a general $d$-dimensional linear FDDE

$$
\begin{aligned}
\left(D_{c}^{\alpha} y\right)(t) & =\int_{-\tau}^{0} y(t+u) A(d u), \quad t \in[0, T], \\
y(t) & =\xi(t), \quad \text { for a.a. } t \in[-\tau, 0), \quad y(0)=\xi_{0},
\end{aligned}
$$

where $D_{c}^{\alpha}$ denotes the Caputo derivative of order $\alpha \in(0,1), A$ is a $\mathbb{R} \times \mathbb{R}$ valued matrix of signed $\sigma$-finite Borel measures, $\tau$ time delay and $((\xi(x)$ : $\left.x \in[-\tau, 0]), \xi_{0}\right)$ is the initial condition.

Using the inverse Laplace transform method we are able to give necessary and sufficient conditions for asymptotic stability of linear FDDEs. It turns out that this property depends on the structure of the measure $A$. If $A$ satisfies $A[-\tau, 0] \neq 0$ and $\operatorname{det} A[-\tau, 0] \neq 0$ then the fundamental solution converges to zero at polynomial rate. In the case $A[-\tau, 0]=0$ we obtain polynomial convergence to the identity matrix.

This paper is structured as follows. In Section 2 we introduce notation, definitions, and preliminary facts which are used throughout this paper. In Section 3 we prove existence and uniqueness results for linear FDDEs. In Section 4 we present the Laplace transform of the fundamental solution $R$ and general solution $y$ and show how $y$ can be expressed in terms of $R$ and the initial conditions $\xi, \xi_{0}$. In the last section we state and prove our main result on asymptotic properties of the fundamental solution.

\section{Fractional Order Delay Differential Equation}

In this section, we present notation, definitions, and recall well-known results about fractional differential equations. For more details the interested reader is referred to the books by Samko et al. ([12], Chapter 2) and Kilbas et al. ([7], Chapter 2).

We first introduce the Riemann-Liouville fractional integral, which is a generalization of the Cauchy formula for repeated integration ([11], section 2.7). Throughout this paper, we assume that $T>0$ and $0 \leq \alpha \leq 1$, which is the case in many applications.

Definition 2.1. Let $f \in L^{1}[0, T]$. The integral

$$
\left(I^{\alpha} f\right)(x):=\frac{1}{\Gamma(\alpha)} \int_{0}^{x}(x-t)^{\alpha-1} f(t) d t, \quad x \in[0, T],
$$

is called the Riemann-Liouville fractional integral of order $\alpha$. For $\alpha=0$ we set $I^{\alpha}=\mathrm{Id}$, the identity operator. 
The fractional derivative operator is the inverse operator of the RiemannLiouville fractional integral:

\section{Definition 2.2.}

1. By $D$ we denote the operator that maps a differentiable function onto its derivative, i.e.,

$$
D f(x):=f^{\prime}(x)
$$

2. For functions $f$ on $[0, T]$ the operator $D^{\alpha}$ defined by

$$
\left(D^{\alpha} f\right)(x):=D\left(I^{1-\alpha} f\right)(x)=\frac{1}{\Gamma(1-\alpha)} \frac{d}{d x} \int_{0}^{x}(x-t)^{-\alpha} f(t) d t, \quad x \in(0, T)
$$

is called Riemann-Liouville fractional derivative of order $\alpha$.

In particular, $D^{0} f=f$ and $D^{1} f=D f$. The following lemma gives a sufficient condition for existence of fractional derivatives:

Lemma 2.1 ([12], Lemma 2.2). Let $f$ be an absolutely continuous function, i.e., $f \in C_{a}[0, T]$, where

$C_{a}[0, T]=\left\{f:[0, T] \rightarrow \mathbb{R}, \exists g \in L^{1}[0, T]: f(x)=f(0)+\int_{0}^{x} g(t) d t, \forall x \in[0, T]\right\}$.

Then $D^{\alpha} f$ exists almost everywhere. Moreover $D^{\alpha} f \in L^{r}(0, T), 1 \leq r<$ $1 / \alpha$ and

$$
\left(D^{\alpha} f\right)(x)=\frac{1}{\Gamma(1-\alpha)}\left[\frac{f(0)}{x^{\alpha}}+\int_{0}^{x}(x-t)^{-\alpha} f^{\prime}(t) d t\right] .
$$

When dealing with fractional differential equations one needs to specify certain initial conditions to guarantee the uniqueness of solution. For the Riemann-Liouville approach values of certain fractional derivatives and integrals are needed ([12], Chapter 42). We shall therefore turn our attention to the Caputo approach, where the values of the function $f$ itself and its integer-order derivatives are specified as initial conditions, [3].

Definition 2.3. The Caputo derivative of order $\alpha \in[0,1]$ is defined via the Riemann-Liouville fractional derivative by

$$
\left(D_{c}^{\alpha} f\right)(x):=\left(D^{\alpha}[f(t)-f(0)]\right)(x), \quad x \in[0, T] .
$$

The Caputo derivative is well-defined for functions for which the RiemannLiouville derivative exists. In particular, it is defined for absolutely integrable functions $f \in C_{a}[0, T]$. 
Theorem 2.1 ([7], Theorem 2.1). If $f \in C_{a}[0, T]$ then the Caputo fractional derivative $D_{c}^{\alpha} f$ exists almost everywhere on $[0, T]$. If $\alpha \notin \mathbb{N}, D_{c}^{\alpha} f$ can be represented by

$$
\left(D_{c}^{\alpha} f\right)(x)=\frac{1}{\Gamma(1-\alpha)} \int_{0}^{x}(x-t)^{-\alpha} f^{\prime}(t) d t=:\left(I^{1-\alpha} D f\right)(x) .
$$

In particular, $D_{c}^{0} f=f$ and $D_{c}^{1} f=f^{\prime}$.

Theorem 2.2 (Properties of the Caputo derivative).

1. Let $f$ be a function with $f \in L^{\infty}(a, b)$. Then

$$
\left(D_{c}^{\alpha} I^{\alpha} f\right)(x)=f(x) \quad \text { for a.a. } x \in(a, b) .
$$

2. Let $f$ be a function with $f \in C([a, b])$. Then

$$
\left(D_{c}^{\alpha} I^{\alpha} f\right)(x)=f(x) \quad \text { for all } x \in[a, b] .
$$

Proof. The proof can be found in [7], Lemma 2.21.

Remark 2.1. The above definitions and results can be generalized to the vector-valued functions, since the integrals and derivatives are taken componentwise.

\section{Existence and Uniqueness of Solutions of linear FDDEs}

Before discussing the questions of existence and uniqueness of solutions of linear FDDEs, we introduce some notation and terminology used in the theory of delay differential equations.

Let $C\left(I, \mathbb{R}^{d}\right)$ denote the set of continuous mappings from an interval $I \subseteq \mathbb{R}$ to $\mathbb{R}^{d}$. The segment $\varphi_{t}$ at time $t \geq 0$ of a function $\varphi \in C\left(I, \mathbb{R}^{d}\right)$ is defined as

$$
\varphi:[-\tau, 0] \rightarrow \mathbb{R}^{d}, \quad \varphi_{t}(u):=\varphi(t+u) .
$$

For a linear, continuous and autonomous operator $f: C\left([-\tau, 0], \mathbb{R}^{d}\right) \rightarrow \mathbb{R}^{d}$ let us consider the following fractional delay differential equation:

$$
\left(D_{c}^{\alpha} y\right)(t)=f\left(y_{t}\right) t \geq 0, \quad y_{0}=\xi,
$$

where $D_{c}^{\alpha}$ denotes the Caputo derivative of order $\alpha \in[0,1], \xi \in C\left([-\tau, 0], \mathbb{R}^{d}\right)$ is the initial condition and $\tau>0$ is the length of the memory. According to the Riesz representation theorem, $f$ can be represented as an integral with 
respect to a $\mathbb{R}^{d} \times \mathbb{R}^{d}$-valued matrix $A=\left(a_{i j}\right), 1 \leq i, j \leq d$, of finite signed Borel-measures $a_{i j}$ on $[-\tau, 0]$ :

$$
f(\varphi)=\int_{-\tau}^{0} \varphi(u) A(d u), \quad \text { for all } \varphi \in C\left([-\tau, 0], \mathbb{R}^{d}\right) .
$$

Hence, in the sequel we consider the following differential equation

$$
\left(D_{c}^{\alpha} y\right)(t)=\int_{-\tau}^{0} y(t+u) A(d u), \quad t \in[0, T], \quad T>0 .
$$

For the equation (3.2) to be well-defined we need to assign values of $y$ for a.a. $t \in[-\tau, 0)$ and for $t=0$ :

$$
y(t)=\xi(t), \quad \text { for a.a. } t \in[-\tau, 0), \quad y(0)=\xi_{0},
$$

where $\xi$ is an $\mathbb{R}^{d}$-valued bounded measurable function on $[-\tau, 0)$, i.e., $\xi \in$ $L^{\infty}\left([-\tau, 0), \mathbb{R}^{d}\right)$ and $\xi_{0} \in \mathbb{R}^{d}$. For continuous initial conditions, we consider $\xi \in C\left([-\tau, 0], \mathbb{R}^{d}\right)$.

Definition 3.1. An $\mathbb{R}^{d}$-valued function $y$ on $[-\tau, T]$ is called a solution of (3.2) with initial condition (3.3) if it is continuous on $[0, T]$, satisfies (3.3) on $[-\tau, 0]$ and

$$
\begin{aligned}
y(t) & =\xi(0)+I^{\alpha}\left(\int_{-\tau}^{0} y(t+u) A(d u)\right) \\
& =\xi(0)+\frac{1}{\Gamma(\alpha)} \int_{0}^{t}(t-s)^{\alpha-1} \int_{-\tau}^{0} y(s+u) A(d u) d s
\end{aligned}
$$

for $t \in[0, T]$.

The following lemma shows that the definition makes sense.

Lemma 3.1. Let $y$ be an $\mathbb{R}^{d}$-valued function on $[-\tau, T]$, continuous on $[0, T]$, satisfying (3.3) on $[-\tau, 0]$.

1. If $y$ is a solution of the integral equation (3.4) with initial condition (3.3) then $y$ solves the differential equation (3.2) for a.a. $t \in[0, \tau)$ and all $t \geq \tau$.

2. If $y$ is a solution of the differential equation (3.2) with initial condition (3.3) then $y$ solves the integral equation (3.4) for all $t \geq 0$.

\section{Remark 3.1.}

Is $\xi$ continuous on $[-\tau, 0)$ and $y$ is the solution of the integral equation, then $y$ solves (3.2) and (3.3) also for $t \in[0, \tau)$, i.e. we obtain an if and only if statement. 
Proof.

1) The function $t \mapsto \int_{-\tau}^{0} y(t+u) A(d u)$ belongs to the class $L^{\infty}[0, \tau)$ and is continuous on $[\tau, T]$.

$$
D_{c}^{\alpha} I^{\alpha} \int_{-\tau}^{0} y(t+u) A(d u) \stackrel{(2.5)}{=} \int_{-\tau}^{0} y(t+u) A(d u)
$$

and for a.a. $t \in[0, \tau)$ and all $t \geq \tau$ it holds that

$$
\begin{aligned}
D_{c}^{\alpha} y(t) & \stackrel{(3.4)}{=} D_{c}^{\alpha}\left(\xi(0)+I_{0}^{\alpha} \int_{-\tau}^{0} y(t+u) A(d u)\right) \\
& =D_{c}^{\alpha} \xi(0)+D_{c}^{\alpha}\left[I_{0}^{\alpha} \int_{-\tau}^{0} y(t+u) A(d u)\right] \\
& =\int_{-\tau}^{0} y(t+u) A(d u) .
\end{aligned}
$$

2) The proof is similar to the proof of Lemma 2.1 of [3].

In the view of Lemma 3.1 we need to prove the existence and the uniqueness of the solution of (3.3) and (3.4). The following theorem is proved by means of the theory of Volterra equations.

Theorem 3.1. Let $\xi$ be an $\mathbb{R}^{d}$-valued measurable bounded function on $[-\tau, 0)$ and $\xi_{0} \in \mathbb{R}^{d}$. There exists a unique function $y:[-\tau, T] \rightarrow \mathbb{R}^{d}$, continuous on $[0, T]$ that satisfies (3.3) and (3.4).

Proof. The space $C\left([0, T], \mathbb{R}^{d}\right)$ is a complete metric space endowed with the supremum metric. On $C\left([0, T], \mathbb{R}^{d}\right)$ we define the linear map $G$ by

$$
(G y)(t)=\left\{\begin{array}{lc}
\xi(0)+\frac{1}{\Gamma(\alpha)} \int_{0}^{t}(t-s)^{\alpha-1} \int_{-\tau}^{0} \xi(s+u) A(d u) d s & t \in[0, \tau] \\
\xi(0)+\frac{1}{\Gamma(\alpha)} \int_{0}^{t}(t-s)^{\alpha-1} \int_{-\tau}^{0} y(s+u) A(d u) d s, & t \in[\tau, T] .
\end{array}\right.
$$

$G$ is continuous: for $t \in[0, \tau]$ it holds that

$$
y(t)=\xi(0)+\int_{\mathbb{R}} g_{t}(s) \int_{-\tau}^{0} \xi(s+u) A(d u) d s,
$$

where $g_{t}(s):=\frac{1}{\Gamma(\alpha)} \mathbf{1}_{\{0 \leq s<t\}}(t-s)^{\alpha-1} \in L^{1}(\mathbb{R}, \mathbb{R})$. There exists a continuous function $g_{t, c}$ with a compact support, satisfying $\left\|g_{t, c}-g_{t}\right\|_{L^{1}} \leq \epsilon$. Moreover, for $\left|t-t^{\prime}\right| \leq \delta$ it holds that $\left\|g_{t, c}-g_{t^{\prime}, c}\right\|_{L^{1}} \leq \epsilon$. Applying the triangle inequality we obtain

$$
\left|y(t)-y\left(t^{\prime}\right)\right| \leq\|A\|\|\xi\|_{\infty} \int_{\mathbb{R}}\left|g_{t}(s)-g_{t^{\prime}}(s)\right| d s \leq 3\|A\|\|\xi\|_{\infty} \epsilon,
$$


where the norm of $A$ is defined by

$$
\|A\|:=\sup _{\|f\|_{\infty}=1}\left\|\int_{-\tau}^{0} f(u) A(d u)\right\|
$$

and the norm on the right hand side denotes the usual operator norm for matrices. The continuity of $y$ for $t \geq \tau$ is shown analogously.

Similar to the proof of Theorem 2.2. in [3] it can be shown by induction that

$$
\left\|G^{j} y-G^{j} z\right\|_{L_{\infty}[0, T]} \leq \frac{\left(\|A\| t^{\alpha}\right)^{j}}{\Gamma(1+\alpha j)}\|y-z\|_{L_{\infty}[0, T]}
$$

holds. Hence, the Fixed Point Theorem of Weissinger ([7], Theorem 1.10) implies the existence of a unique fixed point $x \in C\left([0, T], \mathbb{R}^{d}\right)$. Using (3.3) we extend $y$ to $[-\tau, T]$. The function $y$ is also right-continuous in 0 , since it holds that

$$
\left|x(t)-\xi_{0}\right| \leq\left|x(t)-\left(G^{n} y_{0}\right)(t)\right|+\left|\left(G^{n} y_{0}\right)(t)-\left(G^{n} y_{0}\right)(0)\right|+\left|\left(G^{n} y_{0}\right)(0)-\xi_{0}\right| \rightarrow 0 .
$$

\section{Representation of Solutions}

We first introduce the notions of the fundamental solution and the characteristic function which play a significant role in the theory of DDEs.

Definition 4.1. A function $R: \mathbb{R} \rightarrow \mathbb{R}^{d \times d}$ is called fundamental solution of (3.3) and (3.4) if its $i^{\text {th }}$ column $R^{i}, i=1, \ldots, d$, satisfies

$$
R^{i}(t)= \begin{cases}0, & t<0 \\ e_{i}, & t=0 \\ e_{i}+\frac{1}{\Gamma(\alpha)} \int_{0}^{t}(t-s)^{\alpha-1} \int_{-\tau}^{0} R^{i}(u+s) A(d u) d s, & t>0\end{cases}
$$

where $\left\{e_{1}, \ldots e_{d}\right\}$ denotes the standard basis of $\mathbb{R}^{d}$.

The characteristic function associated with equation (3.3) and (3.4) is defined by

$\chi_{A}(z)=z\left(E_{d}-z^{-\alpha} \int_{-\tau}^{0} e^{z u} A(d u)\right)=\frac{z^{\alpha} E_{d}-\int_{-\tau}^{0} e^{z u} A(d u)}{z^{\alpha-1}}, \quad z \in \mathbb{C} \backslash\{0\}$,

where $E_{d}$ denotes the identity matrix for $\mathbb{R}^{d} \times \mathbb{R}^{d}$.

Remark 4.1. To define the Laplace transform uniquely, we take the following branch of the function $z^{\alpha}$ :

$$
z^{\alpha}:= \begin{cases}|z|^{\alpha} e^{i \alpha \arg z}, & -\pi<\arg z<\varphi_{0} \\ |z|^{\alpha} e^{i \alpha \arg z-2 \pi i \alpha}, & \varphi_{0}<\arg z \leq \pi\end{cases}
$$


for $\varphi_{0} \in(-\pi, \pi),\left|\varphi_{0}\right| \geq \pi / 2$. The proof of the theorem 5.1 and remark 5.1 will give the justification for taking this particular branch instead of the principle branch of $z^{\alpha}$.

Lemma 4.1. For a finite signed Borel measure $A$ the fundamental solution $R$ exists and is unique. Its Laplace transform is defined for $z \in \mathbb{C}$, with $\operatorname{Re} z>\|A\|^{1 / \alpha}$ and is given by

$$
L[R](z)=\left(\chi_{A}(z)\right)^{-1} .
$$

Proof. On the Banach space $C\left([0, T], \mathbb{R}^{d \times d}\right)$ we define the norm

$$
\|f\|=\sup _{t \in[0, T]} \sup _{\|x\|=1}\|f(t) x\| .
$$

The generalization of Theorem 3.1 to matrix-valued functions $f$ is immediate. Existence and the form of the Laplace transform follows from the next result.

Theorem 4.1. Let $y$ be a solution of (3.3) with initial condition (3.4). Its Laplace transform exists for all $z$ with $\operatorname{Re} z>\|A\|^{1 / \alpha}$ and is given by

$L[y](z)=\left[\xi_{0} z^{\alpha-1}+\int_{-\tau}^{0} e^{z u}\left(\int_{u}^{0} e^{-z t} \xi(t) d t\right) A(d u)\right]\left[z^{\alpha} E_{d}-\int_{-\tau}^{0} e^{-z u} A(d u)\right]^{-1}$.

Proof. With $c=\|\xi\|_{\infty}+\left|\xi_{0}\right|$ it holds that:

$$
\begin{aligned}
\sup _{0 \leq w \leq t}|y(w)| & \leq c+\frac{1}{\Gamma(\alpha)} \sup _{0 \leq w \leq t} \int_{0}^{w}(w-s)^{\alpha-1}\left|\int_{-\tau}^{0} y(u+s) A(d u)\right| d s \\
& \leq c+\frac{\|A\|}{\Gamma(\alpha)} \sup _{0 \leq w \leq t} \int_{0}^{w}(w-s)^{\alpha-1} \sup _{-\tau \leq u \leq s}|y(u)| d s .
\end{aligned}
$$

Moreover, $\sup _{-\tau \leq w \leq 0}|y(w)|=c$ and we obtain

$$
\begin{aligned}
\sup _{-\tau \leq w \leq t}|y(w)| & \leq c+\frac{\|A\|}{\Gamma(\alpha)} \sup _{0 \leq w \leq t} \underbrace{\int_{0}^{w}(w-s)^{\alpha-1} \sup _{-\tau \leq u \leq s}|y(u)| d s}_{\text {increasing in } w} \\
& =c+\frac{\|A\|}{\Gamma(\alpha)} \int_{0}^{t}(t-s)^{\alpha-1} \sup _{-\tau \leq u \leq s}|y(u)| d s .
\end{aligned}
$$

We shall use a Gronwall-type result (Lemma 4.3 in [2]) to obtain a bound for $y$ :

$$
\sup _{-\tau \leq w \leq t}|y(w)| \leq c E_{\alpha}\left(\|A\| t^{\alpha}\right) .
$$


Here, $E_{\alpha}(z)$ denotes the Mittag-Leffler function

$$
E_{\alpha}(z):=\sum_{k=0}^{\infty} \frac{z^{k}}{\Gamma(\alpha k+1)} .
$$

The Mittag-Leffler function has the following asymptotic properties for $0<$ $\alpha<2$, see e.g. [4], Chapter 18.1, (10):

$$
E_{\alpha}(x) \sim \frac{1}{\alpha} \exp \left\{x^{1 / \alpha}\right\}+O\left(x^{-1}\right), \quad \text { for } x \rightarrow \infty .
$$

Hence,

$$
E_{\alpha}\left(\|A\| x^{\alpha}\right) \sim \exp \left\{\|A\|^{1 / \alpha} x\right\}+O\left(x^{-\alpha}\right), \quad \text { for } x \rightarrow \infty .
$$

We obtain from (4.3) and (4.5)

$$
\sup _{-\tau \leq w \leq t}|y(w)| \leq c E_{\alpha}\left(\|A\| t^{\alpha}\right) \leq \tilde{c} \exp \left\{\|A\|^{1 / \alpha} t\right\} .
$$

In particular, the solution $y$ and its integral with respect to the measure $A$ are exponentially bounded: for all $t \geq 0$ it holds that

$$
\begin{aligned}
& \exp \left\{-\|A\|^{1 / \alpha} t\right\}|y(t)| \leq M, \\
& \exp \left\{-\|A\|^{1 / \alpha} t\right\}\left|\int_{-\tau}^{0} y(u+t) A(d u)\right| \\
& \leq\|A\| \exp \left\{-\|A\|^{1 / \alpha} t\right\} \sup _{-\tau \leq s \leq t}|y(s)| \leq M^{\prime}
\end{aligned}
$$

for some constants $M, M^{\prime}$. Hence, the Laplace transform of $y$ and the integral exist for all $z$ with $\operatorname{Re} z>\|A\|^{1 / \alpha}$. It holds that

$$
\begin{aligned}
& L\left[\int_{-\tau}^{0} y(u+\bullet) A(d u)\right](z)=\int_{0}^{\infty} e^{-z t} \int_{-\tau}^{0} y(u+t) A(d u) d t \\
& =\int_{-\tau}^{0} \int_{0}^{\infty} e^{-z t} y(u+t) d t A(d u) \\
& =\int_{-\tau}^{0} \int_{u}^{\infty} e^{-z(t-u)} y(t) d t A(d u) \\
& =\int_{-\tau}^{0} e^{z u}\left(\int_{u}^{0} e^{-z t} \xi(t) d t+\int_{0}^{\infty} e^{-z t} y(t) d t\right) A(d u) \\
& =\int_{-\tau}^{0} e^{z u}\left(\int_{u}^{0} e^{-z t} \xi(t) d t+L[y](z)\right) A(d u) .
\end{aligned}
$$

Let $g: \mathbb{R}_{+} \rightarrow \mathbb{R}$ be the function defined by $g(x):=\frac{x^{\alpha-1}}{\Gamma(\alpha)}$. For $z$ with $\operatorname{Re} z>0$ its Laplace transform is given by $L[g](z)=\frac{1}{z^{\alpha}}$. Applying the 
Fubini theorem we obtain:

$$
\begin{aligned}
L[y](z) & =\frac{\xi_{0}}{z}+\frac{1}{\Gamma(\alpha)} \int_{0}^{\infty} e^{-z t} \underbrace{\int_{0}^{t}(t-s)^{\alpha-1} \int_{-\tau}^{0} y(s+u) A(d u) d s}_{\text {convolution of } g \text { and } \int_{-\tau}^{0} y(u+x) A(d u)} d t \\
& =\frac{\xi_{0}}{z}+\frac{1}{z^{\alpha}} L\left[\int_{-\tau}^{0} y(u+\bullet) A(d u)\right](z) \\
& \stackrel{(4.10)}{=} \frac{\xi_{0}}{z}+\frac{1}{z^{\alpha}}\left(\int_{-\tau}^{0} e^{z u}\left(\int_{u}^{0} e^{-z t} \xi(t) d t+L[y](z)\right) A(d u)\right)
\end{aligned}
$$

and therefore

$L[y](z)\left(E_{d}-\frac{1}{z^{\alpha}} \int_{-\tau}^{0} e^{z u} A(d u)\right)=\frac{\xi_{0}}{z}+\frac{1}{z^{\alpha}}\left(\int_{-\tau}^{0} e^{z u}\left(\int_{u}^{0} e^{-z t} \xi(t) d t\right) A(d u)\right)$.

Hence,

$$
\begin{aligned}
& L[y](z)=\left[\frac{\xi_{0}}{z}+\frac{1}{z^{\alpha}}\left(\int_{-\tau}^{0} e^{z u}\left(\int_{u}^{0} e^{-z t} \xi(t) d t\right) A(d u)\right)\right]\left[E_{d}-\frac{1}{z^{\alpha}} \int_{-\tau}^{0} e^{z u} A(d u)\right]^{-1} \\
& =\left[\xi_{0} z^{\alpha-1}+\left(\int_{-\tau}^{0} e^{z u}\left(\int_{u}^{0} e^{-z t} \xi(t) d t\right) A(d u)\right)\right]\left[z^{\alpha} E_{d}-\int_{-\tau}^{0} e^{z u} A(d u)\right]^{-1} \\
& =\left[\xi_{0}+z^{1-\alpha}\left(\int_{-\tau}^{0} e^{z u}\left(\int_{u}^{0} e^{-z t} \xi(t) d t\right) A(d u)\right)\right]\left[z\left(E_{d}-z^{1-\alpha} \int_{-\tau}^{0} e^{z u} A(d u)\right)\right]^{-1} .
\end{aligned}
$$

Remark 4.2. The Laplace transform of the fundamental solution $R$ exists for all $z$ with $\operatorname{Re} z>\|A\|^{1 / \alpha}$ and is given by

$$
\chi_{A}(z):=z\left(E_{d}-z^{-\alpha} \int_{-\tau}^{0} e^{z u} A(d u)\right)=\frac{z^{\alpha} E_{d}-\int_{-\tau}^{0} e^{z u} A(d u)}{z^{\alpha-1}} .
$$

Proof. Again, the steps of the Theorem 4.1 can be extended to matrix-valued functions.

Lemma 4.2. The solution of $y$ of (3.3) and (3.4) can be represented in terms of the fundamental solution $R$ and the initial condition $\left(\xi, \xi_{0}\right)$ :

$$
\begin{aligned}
y(t) & =\xi_{0} R(t)+\frac{d}{d t} \int_{-\tau}^{0}\left(\int_{u}^{0} \frac{1}{\Gamma(\alpha)} \int_{0}^{t}(t-s)^{\alpha-1} R(s+u-v) d s \xi(v) d v\right) A(d u) \\
& =\xi_{0} R(t)+D_{c}^{1-\alpha} \int_{-\tau}^{0}\left(\int_{u}^{0} R(t+u-v) \xi(v) d v\right) A(d u)
\end{aligned}
$$


Proof. We show that the Laplace transforms of the left and the right hand side of (4.11) coincide.

$$
\begin{aligned}
& L[y](z)-\xi(0) L[R](z) \\
& =L[R](z)\left(z^{1-\alpha} \int_{-\tau}^{0} e^{z u} \int_{u}^{0} e^{-z t} \xi(t) d t A(d u)\right) \\
& =z^{1-\alpha} L[R](z) \int_{-\tau}^{0} \int_{u}^{0} e^{-z(-u+t)} \xi(t) d t A(d u) \\
& =z^{1-\alpha} \int_{-\tau}^{0} \int_{u}^{0} \int_{0}^{\infty} e^{-z(x-u+t)} R(x) d x \xi(t) d t A(d u) \\
& =z^{1-\alpha} \int_{-\tau}^{0} \int_{u}^{0} \int_{t-u}^{\infty} e^{-z x} R(x+u-t) d x \xi(t) d t A(d u) \\
& =z^{1-\alpha} \int_{-\tau}^{0} \int_{u}^{0} \int_{0}^{\infty} e^{-z x} R(x+u-t) d x \xi(t) d t A(d u) \\
& \left(\text { Laplace transform of the convolution of } \frac{1}{\Gamma(\alpha)} x^{\alpha-1} \text { and } R(\bullet+u-t)\right) \\
& =z \int_{-\tau}^{0} \int_{u}^{0} \int_{0}^{\infty} e^{-z x} \int_{0}^{x} \frac{(x-s)^{\alpha-1}}{\Gamma(\alpha)} R(s+u-t) d s d x \xi(t) d t A(d u) .
\end{aligned}
$$

This is the Laplace transform of the right hand side, since the Laplace transform of a derivative of a function is given by $L\left[\frac{d}{d x} f\right](z)=z L[f](z)-$ $f(0)$.

\section{Asymptotic Properties of the Solution}

We show that the inverse Laplace transform of $\chi_{A}^{-1}$ can be expressed in terms of generalised exponentials by the residue theorem of complex analysis. This representation allows us to study the asymptotic properties of the fractional delay differential equations. We first investigate the set of zeros of the characteristic function. Let

$$
\begin{aligned}
N_{A} & =\left\{z \in \mathbb{C} \backslash\{0\}: \operatorname{det} \chi_{A}(z)=0\right\}, \\
v_{0} & =\max \left\{\operatorname{Re}(z): z \in N_{A}\right\}, \\
v_{n} & =\max \left\{\operatorname{Re}(z): z \in N_{A}, \operatorname{Re} z<v_{n-1}\right\}, \\
N_{n} & =N_{A} \cap\left\{z \in \mathbb{C}: \operatorname{Re} z \geq v_{n}\right\} .
\end{aligned}
$$

Lemma 5.1. The set $N_{A}$ has no accumulation points in $\mathbb{C}$. Moreover, for all $z \in N_{A}$ it holds that:

$$
|z| \leq\|A\|^{1 / \alpha} \quad \text { or } \quad|z| e^{\tau \operatorname{Re} z / \alpha} \leq\|A\|^{1 / \alpha} .
$$

The last inequality guarantees that there exist at most finitely many $z \in N_{A}$ with $\operatorname{Re} z \geq \rho$ for any $\rho \in \mathbb{R}$. In particular, the above quantities are well-defined. 
Proof. The function $\chi_{A}$ is holomorphic on $\mathbb{C} \backslash\left(\mathbb{R}_{-} \cup\{0\}\right)$, hence there are no accumulation points of $N_{A}$ in this set.

Suppose that 0 is an accumulation point of $N_{A}$. Then there exists a sequence $\left(z_{n}\right)_{n \in \mathbb{N}},\left|z_{n}\right|>0, z_{n} \rightarrow 0$, such that $\operatorname{det} \tilde{\chi}_{A}\left(z_{n}\right)=0$, where

$$
\tilde{\chi}_{A}(z):=z^{\alpha} E_{d}-\int_{-\tau}^{0} e^{z u} A(d u) .
$$

We apply the Taylor series expansion to the integral. Since $A$ is a finite measure and the function $e^{z u}$ is uniformly bounded for $|z| \leq 1$ on $[-\tau, 0]$, it holds that

$$
\int_{-\tau}^{0} e^{z u} A(d u)=\sum_{k=0}^{\infty} z^{k} \int_{-\tau}^{0} \frac{u^{k}}{k !} A(d u), \quad|z|<1 .
$$

Hence, applying the Leibniz formula for the determinant, we obtain

$$
\operatorname{det} \tilde{\chi}_{A}\left(z_{n}\right)=(-1)^{d}\left((-1)^{d} z_{n}^{d \alpha}+z^{(d-1) \alpha} \sum_{k=0}^{\infty} z_{n}^{k} f_{k}^{d-1}+\ldots+\sum_{k=0}^{\infty} z_{n}^{k} f_{k}^{0}\right),
$$

where

$$
\begin{aligned}
\sum_{l=0}^{\infty} z_{n}^{l} f_{l}^{i} & =\sum_{\substack{\pi \in S_{d} \\
\{j: \pi(j)=j\}=i}} \operatorname{sgn}(\pi)(-1)^{i} \prod_{\{j: \pi(j) \neq j\}} \sum_{l=0}^{\infty} z^{l} \int_{-\tau}^{0} \frac{u^{l}}{l !} A_{j \pi(j)}(d u) \\
& +\sum_{\substack{K \subseteq\{1, \ldots, n\} \\
|K|=d-i}}(-1)^{i} \prod_{j \in K} \sum_{l=0}^{\infty} z^{l} \int_{-\tau}^{0} \frac{u^{l}}{l !} A_{j j}(d u), \quad 0<i<d,
\end{aligned}
$$

and

$$
\sum_{l=0}^{\infty} z_{n}^{l} f_{l}^{0}=\operatorname{det}\left(\sum_{k=0}^{\infty} z^{k} \int_{-\tau}^{0} \frac{u^{k}}{k !} A(d u)\right) .
$$

The series $\sum_{l=0}^{\infty} z_{n}^{l} f_{l}^{i}$ is absolutely convergent, since it is a finite sum of Cauchy products of absolutely convergent series. Hence, $\operatorname{det} \chi_{A}\left(z_{n}\right)$ is also absolutely convergent. Let $f_{\beta_{0}} z^{\beta_{0}}$ be the term in the expansion of $\chi_{A}\left(z_{n}\right)$ satisfying $\beta_{0}=\min \left\{\beta \in \mathbb{R}_{+} \cup\{0\}: f_{\beta} z_{n}^{\beta} \neq 0\right\}$. Then

$$
\left|\operatorname{det} \tilde{\chi}_{A}\left(z_{n}\right)\right| \geq \frac{1}{2}\left|f_{\beta_{0}} z_{n}^{\beta_{0}}\right| \neq 0 \quad \text { for all } n \text { sufficiently large. }
$$

We obtain a contradiction. Since we are considering only one branch of $z^{\alpha}$, the function $\chi_{A}(z)$ is discontinuous on $I=\left\{z \in \mathbb{C}: z=r e^{i \varphi_{0}}, 0<r<\right.$ $\left.\delta / \cos \varphi_{0}\right\}$, but $N_{A}$ has also no accumulation points there. Let

$$
f(z):= \begin{cases}|z|^{\alpha} e^{i \alpha \arg z}, & -\pi<\arg z<\varphi_{1} \\ |z|^{\alpha} e^{i \alpha \arg z-2 \pi i \alpha}, & \varphi_{1}<\arg z \leq \pi\end{cases}
$$


for a $\varphi_{1}<\varphi_{0} . f(z)$ is holomorphic on $I$ and $f(z) \equiv z^{\alpha}$ for $\varphi_{0}<\arg z \leq$ $\pi$. Hence there exists no sequence $z_{n} \rightarrow z_{0} \in I$ with $\left(z_{n}\right)_{n \in \mathbb{N}} \subseteq N_{A}$ and $\arg z_{n} \downarrow \varphi_{0}$. Similarly one can show the same result for a sequence $z_{n}$ with $\arg z_{n} \uparrow \varphi_{0}$.

In order to show the bounds in (5.1), we use the Neumann series argument. It yields that

$$
E_{d}-\frac{1}{z^{\alpha}} \int_{-\tau}^{0} e^{z u} A(d u)
$$

is invertible if $\left\|\frac{1}{z^{\alpha}} \int_{-\tau}^{0} e^{z u} A(d u)\right\|<1$, i.e.,

$$
|z|^{\alpha} \leq\left\|\int_{-\tau}^{0} e^{z u} A(d u)\right\| \leq\|A\|\left(1 \vee e^{-\tau \operatorname{Re} z}\right) .
$$

Therefore, for all $z \in N_{A}$ it holds that $|z|^{\alpha} \leq\|A\|\left(1 \vee e^{-\tau \operatorname{Re} z}\right)$. If there were infinitely many zeros with $\operatorname{Re} z \geq \rho$ for a $\rho \in \mathbb{R}$, then this would imply $\operatorname{Re} z \rightarrow \infty$ or $|\operatorname{Im} z| \rightarrow \infty$ and hence $|z| e^{\tau \operatorname{Re} z} \rightarrow \infty$.

Theorem 5.1. Let $A$ be a $\mathbb{R}^{d} \times \mathbb{R}^{d}$-valued matrix of finite signed Borelmeasures satisfying one of the following two conditions:

(C1) $A[-\tau, 0] \neq 0$ and $\operatorname{det} A[-\tau, 0] \neq 0$,

(C2) $A[-\tau, 0]=0$.

For all $n \in \mathbb{N}$ and any $\delta \in\left(v_{n+1}, v_{n}\right)$ the fundamental solution can be represented as the following sum:

$$
R(t)=\sum_{\lambda \in N_{n}} \operatorname{Res}_{\lambda=z}\left(\frac{1}{\chi_{A}(\lambda)} e^{\lambda t}\right)+g(t),
$$

where the function $g$ satisfies

$$
g(t)= \begin{cases}O\left(e^{\delta t}\right), & \text { if } \delta>0 \\ O\left(t^{-\alpha}\right), & \text { if } \delta<0 \text { and } A \text { satisfies }(C 1), \\ E_{d}+O\left(t^{-k_{0}+\alpha}\right), & \text { if } \delta<0 \text { and } A \text { satisfies (C2), }\end{cases}
$$

where

$$
k_{0}=\min \left\{k \in \mathbb{N}: \int_{-\tau}^{0} u^{k} / k ! A(d u) \neq 0\right\} .
$$

Proof. Case 1: $\delta<0$.

We integrate the function $\chi_{\lambda}^{-1}(z) e^{z t}$ along the keyhole contour $\gamma=\gamma_{1} \cup \ldots \cup$ $\gamma_{8}$, as shown in Figure 1 , with vertices $(\rho, K),(\rho,-K),(\delta,-K)$ and $(\delta, K)$, where $\rho>\|A\|^{1 / \alpha}$. Due to the bounds in (5.1) and the fact that there are no accumulation points of $N_{A}$ on $\mathbb{C}$ there are only finitely many zeros of 
$\operatorname{det} \chi_{A}$ inside the contour, independent of how large $K$ and how small $\epsilon$ are chosen. We can also a find a $\varphi_{0} \in(-\pi, \pi],\left|\varphi_{0}\right| \geq \pi / 2$ such that

$$
\begin{array}{r}
\left\{z=e^{i \varphi}:\left(\varphi_{0}-\eta<\varphi<\varphi_{0} \text { or }-2 \pi+\left(\varphi_{0}+\eta\right)<\varphi<-2 \pi+\varphi_{0}\right),\right. \\
r \cos \varphi \geq \delta, r>0\} \cap N_{A}=\emptyset
\end{array}
$$

for all $\eta$ sufficiently small. We consider the function $z^{\alpha}$ with branch cut at $\varphi=\varphi_{0}$. 


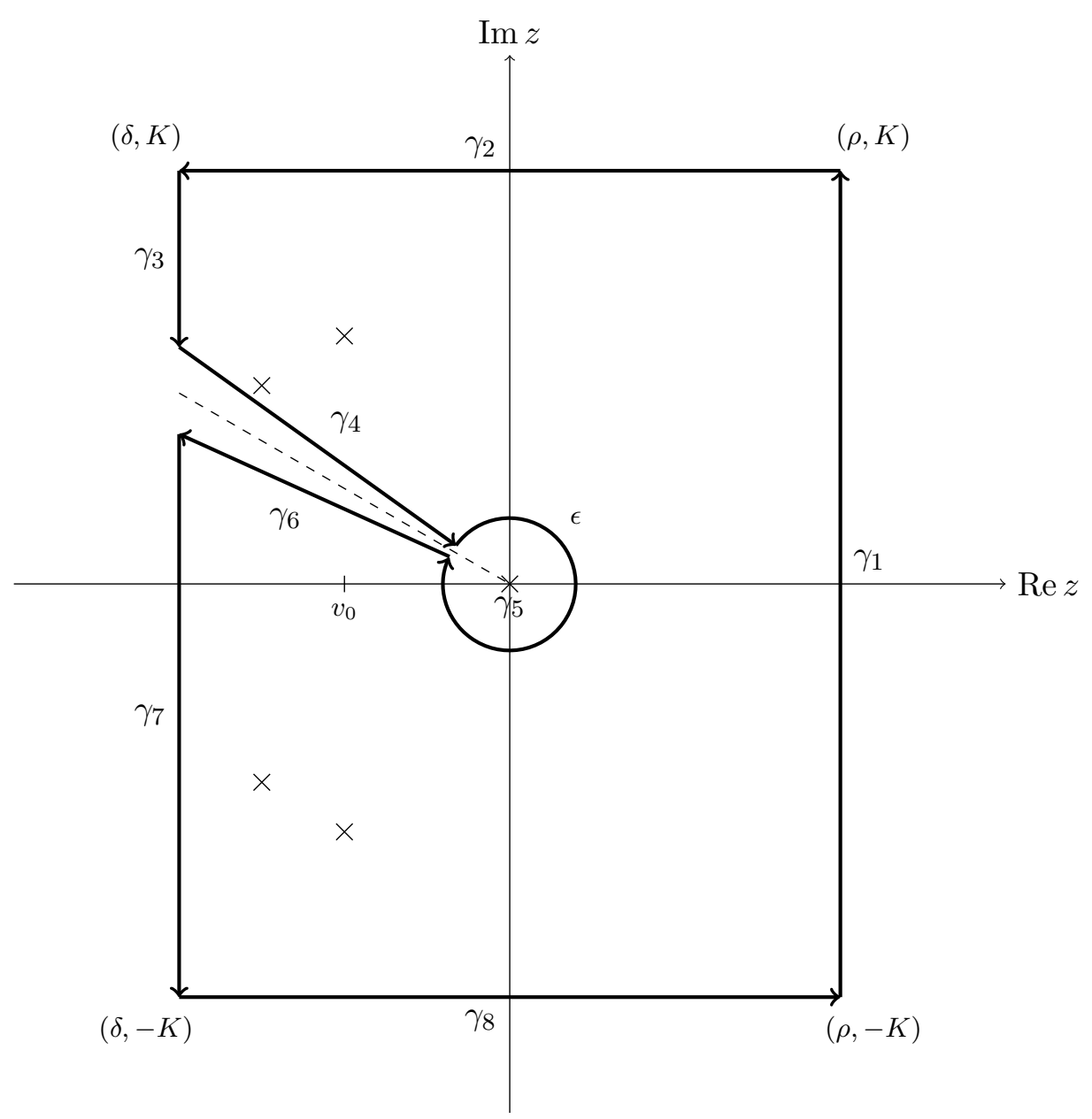

Figure 1: Contour integration of $\chi_{\lambda}^{-1}(z) e^{z t}$. 
The residue theorem yields for every fixed $t$

$$
\left.\frac{1}{2 \pi i} \int_{\gamma}\left(\chi_{A}(z)\right)^{-1} e^{z t} d z=\sum_{s \in N_{n}} \operatorname{Res}_{z=s}\left(\chi_{A}(z)\right)^{-1} e^{z t}\right) .
$$

Since we chose $\rho$ to satisfy $\rho>\|A\|^{1 / \alpha}$, the integral along $\gamma_{1}$ tends to the fundamental solution as $K \rightarrow \infty$ :

$$
R(t)=\frac{1}{2 \pi i} \lim _{K \rightarrow \infty} \int_{\gamma_{1}}\left(\chi_{A}(z)\right)^{-1} e^{z t} d z .
$$

We show that the path integrals over $\gamma_{2}$ and $\gamma_{8}$ vanish as $K \rightarrow \infty$, the limits of the integrals over $\gamma_{3}$ and $\gamma_{7}$ are decaying exponentially for $t \rightarrow \infty$, the limits of the path integrals over $\gamma_{6}$ and $\gamma_{4}$ decay polynomially in $t$ for $\eta \rightarrow 0$ and $\epsilon \rightarrow 0$. The integral over $\gamma_{5}$ depends on wherever $A$ satisfies the condition $(\mathrm{C} 1)$ or $(\mathrm{C} 2)$. In the first case, it vanishes, in the latter case it converges polynomially in $t$ to the identity matrix.

Path integrals over $\gamma_{2}$ and $\gamma_{8}$

Let us consider the integral along $\gamma_{8}$.

$$
I_{8}:=\frac{1}{2 \pi i} \int_{\gamma_{8}} \chi_{A}(z)^{-1} e^{z t} d z .
$$

From the inequality (5.2) we know that $\chi_{A}$ is invertible for $K^{\alpha}>(1 \mathrm{~V}$ $\left.e^{-\tau \delta}\right)\|A\|$. The Neumann series argument yields the following bound of the inverse function if we choose $K$ large enough:

$$
\begin{aligned}
\left\|\chi_{A}(z)^{-1}\right\| & =\left\|z^{-1}\left(E_{d}-z^{-\alpha} \int_{-\tau}^{0} e^{z u} A(d u)\right)^{-1}\right\| \\
& \leq|z|^{-1} \frac{1}{1-\left\|z^{-\alpha} \int_{-\tau}^{0} e^{z u} A(d u)\right\|} \\
& \leq \frac{|z|^{\alpha-1}}{|z|^{\alpha}-\left(1 \vee e^{-\tau \delta}\right)\|A\|} \\
& \leq \frac{K^{\alpha-1}}{K^{\alpha}-\left(1 \vee e^{-\tau \delta}\right)\|A\|}
\end{aligned}
$$

Therefore,

$$
\begin{aligned}
\left\|I_{8}\right\| & \leq \int_{\delta}^{\rho}\left\|\left(\chi_{A}(x-i K)\right)^{-1}\right\| e^{x t} d x \leq \frac{K^{\alpha-1}}{K^{\alpha}-\left(1 \vee e^{-\tau \delta}\right)\|A\|} \int_{\delta}^{\rho} e^{x t} d x \\
& =\frac{K^{\alpha-1}}{K^{\alpha}-\left(1 \vee e^{-\tau \delta}\right)\|A\|} \frac{e^{\rho t}-e^{\delta t}}{t} \rightarrow 0 \quad \text { for } K \rightarrow \infty .
\end{aligned}
$$

The same arguments hold for the integral along $\gamma_{2}$. 


\section{Integral along $\gamma_{5}$}

Let $z=\epsilon e^{i \vartheta}$, then $d z=i \epsilon e^{i \vartheta} d \vartheta=i z d \vartheta$.

$$
\begin{aligned}
I_{5} & :=\frac{1}{2 \pi i} \int_{\gamma_{5}} \frac{1}{z}\left(E_{d}-z^{-\alpha} \int_{-\tau}^{0} e^{z u} A(d u)\right)^{-1} e^{z t} d z \\
& =\frac{1}{2 \pi i} \int_{\pi}^{-\pi}\left(E_{d}-z^{-\alpha} \int_{-\tau}^{0} e^{z u} A(d u)\right)^{-1} e^{z t} i d \vartheta
\end{aligned}
$$

We distinguish two cases. Let $A[-\tau, 0] \neq 0$ and $\operatorname{det} A[-\tau, 0] \neq 0$. Then $z^{\alpha} E_{d}-\int_{-\tau}^{0} e^{z u} A(d u) \rightarrow-A[-\tau, 0]$ for $\epsilon \rightarrow 0$. The dominated convergence theorem yields for every fixed $t$

$$
\begin{aligned}
\left\|I_{5}\right\| & =\left\|\frac{1}{2 \pi i} \int_{\pi}^{-\pi} z^{\alpha}\left(z^{\alpha} E_{d}-\int_{-\tau}^{0} e^{z u} A(d u)\right)^{-1} e^{z t} i d \vartheta\right\| \\
& \leq c \epsilon^{\alpha}\left\|(A[-\tau, 0])^{-1}\right\| e^{\epsilon \cos t} \rightarrow 0 \quad \text { for } \epsilon \rightarrow 0
\end{aligned}
$$

with a constant $c$ independent of $t$. Let $A[-\tau, 0]=0$. The first term in the Taylor series expansion of the integral vanishes and it holds that $\int_{-\tau}^{0} e^{z u} A(d u)=\sum_{k=1}^{\infty} z^{k} \int_{-\tau}^{0} \frac{u^{k}}{k !} A(d u)$ and

$$
E_{d}-z^{-\alpha} \int_{-\tau}^{0} e^{z u} A(d u) \rightarrow E_{d} \quad \text { for } \epsilon \rightarrow 0 .
$$

Applying again the dominated convergence theorem we obtain

$$
I_{5} \rightarrow \frac{1}{2 \pi i} \int_{\pi}^{-\pi} E_{d} i d \vartheta=-E_{d} .
$$

\section{Integrals along the paths $\gamma_{3}$ and $\gamma_{7}$}

Let $z=\delta+i y$. with $y_{1}=\delta \tan \left(\varphi_{0}-\eta\right), y_{2}=\delta \tan \left(\varphi_{0}+\eta\right)$ it holds that

$$
\begin{aligned}
I_{3,7} & =\frac{1}{2 \pi i} \int_{\gamma_{3} \cup \gamma_{7}} \chi_{A}^{-1}(z) e^{z t} d z \\
& =\frac{1}{2 \pi} \int_{K}^{y_{1}} \chi_{A}^{-1}(z) e^{z t} d y+\frac{1}{2 \pi} \int_{y_{2}}^{-K} \chi_{A}^{-1}(z) e^{z t} d y \\
& =\frac{1}{2 \pi} \int_{K}^{y_{1}}\left(\chi_{A}^{-1}(z)-\left(z-v_{n+1}\right)^{-1} E_{d}\right) e^{z t} d y \\
& +\frac{1}{2 \pi} \int_{y_{2}}^{-K}\left(\chi_{A}^{-1}(z)-\left(z-v_{n+1}\right)^{-1} E_{d}\right) e^{z t} d y \\
& +\frac{1}{2 \pi} \int_{K}^{y_{1}}\left(z-v_{n+1}\right)^{-1} E_{d} e^{z t} d y+\frac{1}{2 \pi} \int_{y_{2}}^{-K}\left(z-v_{n+1}\right)^{-1} E_{d} e^{z t} d y
\end{aligned}
$$


It holds that

$$
\begin{aligned}
& \lim _{K \rightarrow \infty} \lim _{\eta \rightarrow 0}\left(\frac{1}{2 \pi} \int_{K}^{y_{1}}\left(z-v_{n+1}\right)^{-1} e^{z t} d y+\frac{1}{2 \pi} \int_{y_{2}}^{-K}\left(v_{n+1}-z\right)^{-1} e^{z t} d y\right) E_{d} \\
& =\left(-\frac{1}{2 \pi} \int_{-\infty}^{\infty}\left(z-v_{n+1}\right)^{-1} e^{z t} d y\right) E_{d}=-e^{v_{n+1} t} E_{d} .
\end{aligned}
$$

The last equality holds due to the fact that the Laplace transform of $x \mapsto e^{a x}$ is equal to $\frac{1}{z-a}$.

Let us now consider the first term in the representation of $I_{3,7}$ :

$$
\begin{aligned}
& \frac{1}{2 \pi} \int_{K}^{y_{1}}\left(\chi_{A}^{-1}(z)-\left(z-v_{n+1}\right)^{-1} E_{d}\right) e^{z t} d y \\
& =\frac{1}{2 \pi} \int_{K}^{y_{1}}\left(\chi_{A}^{-1}(z)\left(z-v_{n+1}\right)^{-1} E_{d}\right) e^{z t}\left(\left(z-v_{n+1}\right) E_{d}-\chi_{A}(z)\right) d y .
\end{aligned}
$$

Let

$$
x=\min \left\{|\delta-\operatorname{Re} z|: z \in N_{A}\right\} .
$$

Then $|\delta+i y-z| \geq x>0$ for all $z \in N_{A}$ and all $y \in \mathbb{R}_{+}$. Hence there exists a constant $c>0$ such that $\left\|\chi_{A}(\delta+i y)\right\| \geq c$ for all $y \geq \delta \tan \varphi_{0}$. The integral $\int_{-\tau}^{0} e^{(\delta+i y) u} A(d u)$ is bounded along the path $\gamma_{3}$ and hence $\left\|\chi_{A}(\delta+i y)\right\|\left\|\left(v_{n+1}-\delta-i y\right) E_{d}\right\|$ behaves asymptotically like $y^{2}$ for $y \rightarrow+\infty$ :

$$
\left\|\chi_{A}(\delta+i y)\right\|\left\|(\delta+i y)-v_{n+1}\right\| \geq c_{1}\left(1+y^{2}\right)
$$

with a constant $c_{1}$ independent of $t$. On the other hand

$$
\left\|\left(z-v_{n+1}\right) E_{d}-\chi_{A}(z)\right\| \leq\left|v_{n+1}\right|+|z|^{1-\alpha}\left\|\int_{-\tau}^{0} e^{z u} A(d u)\right\| \leq c_{2}\left(1+y^{1-\alpha}\right) .
$$

Hence

$$
\begin{aligned}
& e^{-\delta t}\left\|\frac{1}{2 \pi} \int_{K}^{y_{1}}\left(\chi_{A}^{-1}(z)-\left(z-v_{n+1}\right)^{-1} E_{d}\right) e^{z t} d y\right\| \\
& \leq e^{-\delta t} \int_{0}^{\infty} \frac{c_{2}\left(1+y^{1-\alpha}\right)}{c_{1}\left(1+y^{2}\right)} e^{\delta t} d y \leq \int_{0}^{\infty} c_{3} \frac{1}{1+y^{1+\alpha}} d y<\infty .
\end{aligned}
$$

The second term in the representation of $I_{3,7}$ is treated analogously. Thus, $\sup _{t>0} e^{-\delta t}\left|I_{3,7}\right|<\infty$, since $e^{\left(v_{n+1}-\delta\right) t}$ is bounded for all $t$.

Integrals along the paths $\gamma_{4}$ and $\gamma_{6}$

We investigate the asymptotic properties of

$$
I_{4,6}:=\frac{1}{2 \pi i} \int_{\gamma_{4} \cup \gamma_{6}} \chi_{A}^{-1}(z) e^{z t} d z
$$

as $\eta$ and $\epsilon$ tend to zero simultaneously. We will need $\eta$ to converge to zero quicker than $\epsilon$ and choose therefore $\eta=\epsilon^{n}, n>k_{0}$, where $k_{0}$ is defined as in (5.3). We show 
$A$ satisfies (C1): $\sup _{t>0} \lim _{\epsilon \rightarrow 0} t^{\alpha} I_{4,6}<\infty$.

$A$ satisfies (C2): $\sup _{t>0} \lim _{\epsilon \rightarrow 0} t^{k_{0}-\alpha} I_{4,6}<\infty$.

On $\gamma_{4}$ and $\gamma_{6}$ we set

$$
\begin{gathered}
z=r e^{i\left(\varphi_{0}-\eta\right)}, \epsilon \leq r \leq \delta / \cos \left(\varphi_{0}-\eta\right), \tilde{z}=r e^{i\left(\varphi_{0}+\eta\right)}, \epsilon \leq r \leq \delta / \cos \left(\varphi_{0}+\eta\right), \\
z^{\alpha}=|z|^{\alpha} e^{i \arg z \alpha}=r^{\alpha} e^{i\left(\varphi_{0}-\eta\right) \alpha}, \tilde{z}^{\alpha}=|\tilde{z}|^{\alpha} e^{i \arg \tilde{z} \alpha-2 \pi i \alpha}=r^{\alpha} e^{i\left(\varphi_{0}+\eta\right) \alpha-2 \pi i \alpha},
\end{gathered}
$$

and write

$$
\begin{aligned}
I_{4,6} & =\frac{1}{2 \pi i} \int_{\delta / \cos \left(\varphi_{0}-\eta\right)}^{\epsilon} \frac{1}{z}\left(E_{d}-z^{-\alpha} \int_{-\tau}^{0} e^{z u} A(d u)\right)^{-1} e^{z t} e^{i\left(\varphi_{0}-\eta\right)} d r \\
& +\frac{1}{2 \pi i} \int_{\epsilon}^{\delta / \cos \left(\varphi_{0}+\eta\right)} \frac{1}{\bar{z}}\left(E_{d}-\tilde{z}^{-\alpha} \int_{-\tau}^{0} e^{\tilde{z} u} A(d u)\right)^{-1} e^{\tilde{z} t} e^{i\left(\varphi_{0}+\eta\right)} d r .
\end{aligned}
$$

We study the behaviour of the integrated functions in the neighbourhood of zero and on the interval $I_{ \pm}=\left[\epsilon_{0}, \delta / \cos \left(\varphi_{0} \pm \eta\right)\right]$ separately: $I_{4,6}=I_{>\epsilon_{0}}+$ $I_{<\epsilon_{0}}$, where

$$
\begin{aligned}
& I_{>\epsilon_{0}}=-\frac{1}{2 \pi i} \int_{I_{-}} \chi_{A}^{-1}(z) e^{z t} e^{i\left(\varphi_{0}-\eta\right)} d r+\frac{1}{2 \pi i} \int_{I_{+}} \chi_{A}^{-1}(\tilde{z}) e^{\tilde{z} t} e^{i\left(\varphi_{0}+\eta\right)} d r, \\
& I_{<\epsilon_{0}}=-\frac{1}{2 \pi i} \int_{\epsilon}^{\epsilon_{0}} \chi_{A}^{-1}(z) e^{z t} e^{i\left(\varphi_{0}-\eta\right)} d r+\frac{1}{2 \pi i} \int_{\epsilon}^{\epsilon_{0}} \chi_{A}^{-1}(\tilde{z}) e^{\tilde{z} t} e^{i\left(\varphi_{0}+\eta\right)} d r .
\end{aligned}
$$

For any fixed $\epsilon_{0}>0$ the norms of the inverse matrices are bounded for $z \in I_{ \pm}$. Moreover, applying the dominated convergence theorem we obtain

$$
\left\|I_{>\epsilon_{0}}\right\| \leq c \int_{\epsilon}^{\delta / \cos \varphi_{0}} e^{r t \cos \varphi_{0}} d r=c \frac{e^{\delta t}-e^{\epsilon t \cos \varphi_{0}}}{t \cos \varphi_{0}},
$$

where $c$ depends only on $\epsilon_{0}$. So, $\left\|I_{>\epsilon_{0}}\right\|$ decays to zero at exponential rate for $t \rightarrow \infty$.

Remark 5.1. Let $\alpha=1 / 2, \tau=\frac{\log 3}{2}$ and

$$
A=\left(\begin{array}{cc}
\mathbf{1}_{\{-\tau\}} & -2 \cdot \mathbf{1}_{\{0\}} \\
2 \cdot \mathbf{1}_{\{0\}} & -\mathbf{1}_{\{-\tau\}}
\end{array}\right) .
$$

It holds that

$$
\operatorname{det} \chi_{A}(z)=z^{1-\alpha}\left(4-3^{z}-z\right) \quad \text { and } \operatorname{det} \chi_{A}(-1)=0 \text {, }
$$

hence the norms of the inverse matrices $\chi_{A}(z), \chi_{A}(\tilde{z})$ would be not necessary bounded in the limit if we chose the negative real line segment for the path integrals $\gamma_{4}$ and $\gamma_{6}$. A $\varphi_{0}$ satisfying (5.4) guarantees the bound in (5.6). 
Let us now consider the behaviour of the integrand for $\epsilon_{0}$ small enough. Let $A$ satisfy (C1). For $\epsilon_{0}$ sufficiently small it holds that

$$
\left\|z^{\alpha} E_{d}-\int_{-\tau}^{0} e^{z u} A(d u)\right\| \rightarrow\|A[-\tau, 0]\| .
$$

Hence, $\left\|\chi_{A}(z)^{-1}\right\|$ behaves like $r^{\alpha-1}$ for $r \leq \epsilon_{0}$. There exists a constant $c>0$, independent of $t$ and $\epsilon_{0}$ such that

$$
\begin{aligned}
\left\|I_{<\epsilon_{0}}\right\| & \leq \int_{0}^{\epsilon_{0}} c r^{\alpha-1} e^{r \cos \varphi_{0} t} d r \\
& =c\left(-\cos \varphi_{0}\right)^{-\alpha} t^{-\alpha}\left(\Gamma(\alpha)-\Gamma\left(\alpha,-t \epsilon_{0} \cos \varphi_{0}\right)\right) \leq c t^{-\alpha},
\end{aligned}
$$

where $\Gamma(\alpha, y)$ is given by

$$
\begin{gathered}
\Gamma(\alpha, y):=\int_{y}^{\infty} x^{\alpha-1} e^{-x} d x, \quad \text { i.e. } \\
\Gamma(\alpha)-\Gamma(\alpha,-t \delta)=\int_{0}^{-t \delta} x^{\alpha-1} e^{-x} d^{x} \rightarrow \Gamma(\alpha), t \rightarrow \infty .
\end{gathered}
$$

Hence, $\sup _{t>0} \lim _{\epsilon \rightarrow 0} t^{\alpha}\left(\left\|I_{<\epsilon_{0}}\right\|+\left\|I_{>\epsilon_{0}}\right\|\right)$ is finite.

More interesting is the case when $A[-\tau, 0]=0$. In this case the norms of the integrals along $\gamma_{4}$ and $\gamma_{6}$ tend to infinity, but they cancel. This is due to the fact that the only terms which include fractional powers of $z$ tend to zero as $\epsilon_{0}$ and $\eta$ tend to zero, so the integrals cancel due to the opposite sign. Let us first consider the integrated function along $\gamma_{4}$.

From (5.5) we have for all $r \leq \epsilon_{0}$, where $\epsilon_{0}$ is chosen to be sufficiently small,

$$
\|\underbrace{\left(E_{d}-z^{-\alpha} \int_{-\tau}^{0} e^{z u} A(d u)\right)}_{A_{1}(z)}-\underbrace{E_{d}\left(1-c_{1} z^{k_{0}-\alpha}\right)}_{B_{1}(z)}\| \leq c_{2} r^{k_{0}+1-\alpha} .
$$

The constants $c_{1}$ and $c_{2}$ are independent of $r$ and $\epsilon$. Hence,

$$
\left\|\frac{A^{-1}(z)}{z}-\frac{B^{-1}(z)}{z}\right\| \leq\left\|A^{-1}(z)\right\|\left\|B^{-1}(z)\right\| c_{2} r^{k_{0}-\alpha} \leq c_{3} r^{k_{0}-\alpha},
$$

since $A^{-1}(z), B^{-1}(z)$ converge to the identity matrix as $z \rightarrow 0$. Same results 
hold also on $\gamma_{6}$ with constants $\tilde{c_{1}}, \tilde{c_{2}}, \tilde{c_{3}}$. Moreover,

$$
\begin{aligned}
\left\|\frac{B^{-1}(z)}{z}-\frac{B^{-1}(\tilde{z})}{\tilde{z}}\right\| & =\left\|E_{d}\left(\frac{1}{z\left(1-c_{1} z^{k_{0}-\alpha}\right)}-\frac{1}{\tilde{z}\left(1-\tilde{c}_{1} \tilde{z}^{k_{0}-\alpha}\right)}\right)\right\| \\
& \leq\left|\frac{e^{i\left(\varphi_{0}+\eta\right)}-e^{i\left(\varphi_{0}-\eta\right)}}{r}\right|+c_{4} r^{k_{0}-\alpha-1} \\
& =\left|\frac{e^{i \varphi_{0}}\left(e^{i \eta}-e^{-i \eta}\right)}{r}\right|+c_{4} r^{k_{0}-\alpha-1} \\
& \left|\frac{2 e^{i \varphi_{0}} \sin \eta}{r}\right|+c_{4} r^{k_{0}-\alpha-1} \\
& \leq c_{5} r^{n-1}+c_{4} r^{k_{0}-\alpha-1} \leq c_{6} r^{k_{0}-\alpha-1} .
\end{aligned}
$$

We obtain

$$
\begin{aligned}
\left\|I_{<\epsilon_{0}}\right\| & \leq \int_{\epsilon}^{\epsilon_{0}}\left\|\frac{A^{-1}(z)}{z}-\frac{A^{-1}(\tilde{z})}{\tilde{z}}\right\| e^{r \cos \varphi_{0} t} d r \\
& \leq \int_{\epsilon}^{\epsilon_{0}}\left(\left\|\frac{A^{-1}(z)}{z}-\frac{B^{-1}(z)}{z}\right\|+\left\|\frac{A^{-1}(\tilde{z})}{\tilde{z}}-\frac{B^{-1}(\tilde{z})}{\tilde{z}}\right\|\right. \\
& \left.+\left\|\frac{B^{-1}(z)}{z}-\frac{B^{-1}(\tilde{z})}{\tilde{z}}\right\|\right) e^{r \cos \varphi_{0} t} d r \\
& \leq \int_{\epsilon}^{\epsilon_{0}}\left(\left(c_{3}+\tilde{c}_{3}\right) r^{k_{0}-\alpha}+c_{6} r^{k_{0}-\alpha-1}\right) e^{r \cos \varphi_{0} t} d r \\
& \stackrel{\epsilon \rightarrow 0}{\longrightarrow} c_{7}\left(-t \cos \varphi_{0}\right)^{\alpha-k_{0}}\left(\Gamma\left(k_{0}-\alpha\right)-\Gamma\left(k_{0}-\alpha,-\epsilon_{0} t \cos \varphi\right)\right) .
\end{aligned}
$$

Case 2: $\delta>0$.

For $\delta>0$ we only need to consider the rectangle contour with vertices with $(\rho, K),(\rho,-K),(\delta,-K)$ and $(\delta, K)$. Again, the integrals along $\gamma_{2}$ and $\gamma_{8}$ vanish as $K \rightarrow \infty$, where the path integral over $(\delta, K) \rightarrow(\delta,-K)$ grows asymptotically at exponential rate $e^{\delta t}$.

Example 5.1. The case where $A[-\tau, 0] \neq 0$, but det $A[-\tau, 0]=0$ is more complicated. Let us consider the following example. Let $d=2$ and

$$
A=\left(\begin{array}{cc}
\mathbf{1}_{\{-\tau\}} & -\mathbf{1}_{\left\{-\tau_{1}\right\}} \\
\mathbf{1}_{\left\{-\tau_{1}\right\}} & -\mathbf{1}_{\{-\tau\}}
\end{array}\right),
$$

where $0<\tau_{1}<\tau$. It holds that $\operatorname{det} A[-\tau, 0]=-1+1=0$.

$$
B(z):=z^{\alpha} E_{d}-\int_{-\tau}^{0} e^{z u} A(d u)=\left(\begin{array}{cc}
z^{\alpha}-e^{-\tau z} & e^{-\tau_{1} z} \\
-e^{-\tau_{1} z} & z^{\alpha}+e^{-\tau z}
\end{array}\right)
$$

and

$$
\operatorname{det} B(z)=z^{2 \alpha}-\operatorname{det} \int_{-\tau}^{0} e^{z u} A(d u)=z^{2 \alpha}-\sum_{k=1}^{\infty} a_{k} z^{k}
$$


with some $a_{k} \in \mathbb{R}$.

$B^{-1}(z)=\frac{1}{\operatorname{det} B(z)} \operatorname{adj} B(z)=\frac{1}{z^{2 \alpha}-\sum_{k=1}^{\infty} a_{k} z^{k}}\left(\begin{array}{cc}z^{\alpha}+e^{-\tau z} & -e^{-\tau_{1} z} \\ +e^{-\tau_{1} z} & z^{\alpha}-e^{-\tau z}\end{array}\right)$.

The adjugate of $B(z)$ converges to a constant non-zero matrix for $z \rightarrow 0$ and $z^{\alpha-1} \frac{1}{\operatorname{det} B(z)}$ behaves like $z^{\beta}$, where $\beta<-1$. Hence, the path integrals over $\gamma_{5}, \gamma_{4}$ and $\gamma_{6}$ diverge, whereas the other integrals tend to zero.

Let us now consider

$$
A=\left(\begin{array}{cc}
\mathbf{1}_{\{-\tau\}} & \mathbf{1}_{\left\{-\tau_{1}\right\}} \\
\mathbf{1}_{\left\{-\tau_{1}\right\}} & \mathbf{1}_{\{-\tau\}}
\end{array}\right)
$$

Then

$$
\operatorname{det} B(z)=z^{2 \alpha}-z^{\alpha}\left(e^{-\tau z}+e^{-\tau z}\right)+\operatorname{det} \int_{-\tau}^{0} e^{z u} A(d u) .
$$

The adjugate of $B(z)$ again converges to a constant non-zero matrix for $z \rightarrow 0$ and $z^{\alpha-1} \frac{1}{\operatorname{det} B(z)}$ behaves like $z^{-1}$. Hence, the path integral over $\gamma_{5}$ converges to the identity matrix and the path integrals $\gamma_{4}$ and $\gamma_{6}$ cancel.

\section{Conclusions}

In the present paper, we study the asymptotic properties of the fundamental solution fractional delay differential equations. First, the results on existence and uniqueness of solutions of FDDEs are proved. Based on the characteristic function and characteristic equation, several interesting properties of the solution are obtained. We are able to present a complete picture of the asymptotic behaviour in a very general setting, which includes many cases studied in literature.

\section{References}

[1] Y. Chen and K. L. Moore. Analytical stability bound for a class of delayed fractional-order dynamic systems. Nonlinear Dynamics, 29:191$200,2002$.

[2] K. Diethelm and N.J. Ford. Multi-order fractional differential equations and their numerical solution. Appl. Math. Comput., 154.

[3] K. Diethelm and N.J. Ford. Analysis of fractional differential equations, 2002 .

[4] A. Erdëlyi et al. Higher Transcendental Functions, Vol III. McGrawHill, New York, Toronto, London, 1953. 
[5] C. Hwang and Y.-C. Cheng. A numerical algorithm for stability testing of fractional delay systems. Automatica, 42:825-831, 2006.

[6] S.M. Verduyn Lunel J.K. Hale. Introduction to Functional Differential Equations. Springer, New York, 1993.

[7] A. A. Kilbas, H. M. Srivastava, and J. J. Trujillo. Theory and Applications of Fractional Differential Equations, Volume 204 (North-Holland Mathematics Studies). Elsevier Science Inc., New York, NY, USA, 2006.

[8] U. Küchler and B. Mensch. On langevins stochastic differential equation extended by a time delayed term. Stochastics and Stochastic Reports, 40:123 - 144, 1991.

[9] M. P. Lazarević. Finite time stability analysis of $p d^{\alpha}$ fractional control of robotic time-delay systems. Mechanics Research Communications, $33(2): 269-279,2006$.

[10] M. P. Lazarević and A. M Spasić. Finite-time stability analysis of fractional order time-delay systems: Gronwall's approach. Mathematical and Computer Modelling, 49(3-4):475 - 481, 2009.

[11] K.B. Oldham and J. Spanier. The Fractional Calculus: Theory and Application of Differentiation and Integration to Arbitrary Order. Academic Press, New York, 1974.

[12] S. Samko, A. Kilbas, and O. Marichev. Fractional Integrals and Derivatives: Theory and Applications. Gordon and Breach, London, 1993.

[13] C. Li W. Deng and J. Lu. Stability analysis of linear fractional differential system with multiple time delays. Nonlinear Dynamics, 48:409-416, 2007.

[14] X. Zhang. Some results of linear fractional order time-delay system. Applied Mathematics and Computation, 197(1):407 - 411, 2008. 